\title{
Die Ruhe nach dem Sturm - Steuerungskonflikte als Hemmnis für institutionellen Wandel in der deutschen Finanzmarktaufsicht
}

\section{Einleitung}

Die jüngste Finanzmarktkrise gilt als die schwerste seit der Weltwirtschaftskrise von 1929. Dennoch fielen die Reaktionen in Bezug auf die Anpassungen der Finanzmarktaufsicht und -regulierung weniger stark aus als zunächst erwartet. Auch in Deutschland blieb das System der Finanzmarktaufsicht weitgehend unverändert, obwohl es während der Krise weitreichende Reformbestrebungen gegeben hatte. Dieser Beitrag, der an eine breite politikwissenschaftliche Forschung zu Fragen der Finanzmarktpolitik anknüpft, widmet sich den Ursachen, die dem Ausbleiben des institutionellen Wandels zugrunde liegen.

Noch immer wird in Wissenschaft und Politik über die Auswirkungen auf die Finanz- und Wirtschaftssysteme diskutiert. Dies findet allerdings nicht mehr öffentlichkeitswirksam statt, da die Verwerfungen auf dem Finanzmarkt von anderen Themen überlagert worden sind. Entsprechend hat die Bundesregierung bereits Anfang 2011 die Krise für beendet erklärt und die Rückkehr der deutschen Wirtschaft auf einen Wachstumspfad in Aussicht gestellt. ${ }^{1}$ Angefangene Vorhaben zur Verbesserung von Finanzmarktregulierung und -aufsicht werden seither nur noch ambitionslos abgearbeitet oder verlaufen im Sande.

$\mathrm{Zu}$ den Reaktionen auf die Krise gehörten in vielen Staaten Konjunkturprogramme, die als Maßnahmen gegen wirtschaftliche Einbrüche gedacht waren. Zudem wurden Staatseingriffe im Finanzsektor als nötig erachtet, um systemgefährdende Zusammenbrüche einzelner Institute wie der Hypo Real Estate zu verhindern. ${ }^{2}$ Die Krise hatte politische Akteure, Medien, Interessengruppen und auch die Finanzbranche selbst scheinbar davon überzeugt, dass nationale und globale Finanzmärkte einer stärkeren Regulierung und einer besseren Beaufsichtigung bedürfen. Die Krise bot also eine Gelegenheit für eine institutionelle Veränderung in der Finanzaufsicht und -regulierung. ${ }^{3}$ Aufsicht und Regulierung werden häufig synonym verwendet, aber sie beschreiben etwas Verschiedenes. In knapper Form lässt sich Regulierung als Setzung von Regeln beschreiben, die die Ziele der Finanzmarktstabilität und des Verbraucherschutzes verfolgen, während Aufsicht die Überwachung und Durchsetzung der Einhaltung dieser Regeln umfasst. ${ }^{4}$

1 Vgl. BMWi 2011.

2 Vgl. Altvater et al. 2010.

3 Vgl. van der Heijden 2011.

4 Vgl. Ward 2002; Denters 2009; Handke 2010 a. 
Der vorliegende Beitrag zeigt am Beispiel der deutschen Finanzmarktpolitik, weshalb die politischen Ankündigungen von Finanzmarktaufsichtsreformen ohne Ergebnis geblieben sind. Ende 2010 hat die Regierungskoalition aus CDU/CSU und FDP beschlossen, den Umbau der Finanzmarktaufsichtsstruktur von der politischen Agenda zu nehmen und das System der Aufsicht im Vorkrisenzustand zu belassen. ${ }^{5}$ Es stellt sich die Frage, weshalb die Gelegenheit nicht genutzt wurde, um die Aufsichtsstrukturen an die neuen Herausforderungen anzupassen. Dabei ist davon auszugehen, dass Verbesserungen von Aufsicht und Regulierung zunächst angekündigt und in Angriff genommen worden sind, um das öffentliche Gut der Systemstabilität der Finanzmärkte zu schützen, dann allerdings aus (partei-)politischen und strategischen Überlegungen fallen gelassen wurden. Hierbei standen sich verschiedene Partikularinteressen hinsichtlich des politischen Einflusses und der Steuerung von Behörden gegenüber. Das Geflecht von Akteuren, das mit seinen unterschiedlichen Steuerungsintentionen Einfluss auf die Ausgestaltung der deutschen Finanzmarktaufsicht genommen hat, bildet hier den Ausgangspunkt für eine Erklärung des ausbleibenden institutionellen Wandels. Unter Steuerung wird zum einen die Möglichkeit verstanden, Ziele vorzugeben, die durch die Regulierungen erreicht werden sollen, zum anderen Wege und Aufsichtsinstrumente einzurichten, mit denen die Ziele durchgesetzt werden können. ${ }^{6}$ Durch die Festlegung von Mindestkapitalanforderungen und weiterer Zulassungsvoraussetzungen für die Aufnahme des Geschäftsbetriebs ${ }^{7}$ von Banken, Versicherungen und Wertpapierhändlern soll die Systemstabilität als öffentliches Gut ebenso erreicht werden wie durch die Überwachung und Durchsetzung dieser Regeln mit dem Instrument der Finanzmarktaufsicht. ${ }^{8}$ Während die Aufgabe, die Regeln zu verändern und in Reaktion auf die Krise anzupassen, sehr schnell auf die Ebene der EU oder der G20 verlagert wurde, verblieb die Gestaltung der Aufsicht weitgehend auf der nationalen Ebene. ${ }^{9}$ Grundsätzlich ist bei Regulierung und Aufsicht davon auszugehen, dass » ̈̈nderungen bei der Aufsicht weniger kosten als Änderungen der Regulierung ",${ }^{10}$ da ein kleineres, überwiegend nationales Akteursset beteiligt ist. Dennoch fand selbst diese vermeintlich einfach zu realisierende Änderung nicht statt. An dieser Tatsache ändert auch die Errichtung von drei neuen EU-Aufsichtsagenturen ${ }^{11}$ nichts, die 2011 aus den bisherigen Ebene-3-Ausschüssen des Lamfalussy-Verfahrens hervorgegangen sind. Die EU-Behörden, die für die Aufsicht über Banken, Versicherungen und Wertpa-

5 Vgl. Dautzenberg et al. 2010.

6 Vgl. Eichhorn et al. 1985, S. 345 f.

7 Beispielsweise $\$ 26 \mathrm{KWG}$; 55 VAG.

8 Vgl. Rude 2008.

9 Vgl. Denters 2009, S. 67; FSB 2009.

10 Ward 2002, S. 36.

11 Im Januar 2011 nahmen die EBA (European Banking Authority), ESMA (European Securities and Markets Authority) und EIOPA (European Insurance and Occupational Pensions Authority) ihre Arbeit auf, die aus den ehemaligen Ebene-3-Kommitees - CEBS, CESR und CEIOPS - des sogenannten Lamfalussy-Verfahrens hervorgegangen sind. 
pierhandel zuständig sind, bleiben auch weiterhin auf die Kooperationsbereitschaft der nationalen Aufsichtsbehörden angewiesen. Europäische Regulierung, wie sie mit Übernahme der Basel III-Vereinbarungen oder der Solvency II-Regeln ${ }^{12}$ manifest wird, strebt die Stabilität der Finanzmärkte an, hat gleichzeitig aber auch den Anspruch, die Entwicklung des europäischen Binnenmarktes voranzutreiben. ${ }^{13}$

So hat die Ankündigung, durch internationale Kooperation bessere und wirksamere Regulierungsregime zu schaffen, kaum zu tatsächlichen Konsequenzen geführt. Vielmehr ist der »Strang, an dem alle Nationen noch vor ein paar Monaten ziehen wollten, ... zum dürren Fädlein geworden « ${ }^{14}$ An den regulatorischen Vorgaben für Finanzunternehmen hat es keine nennenswerten Veränderungen gegeben, und selbst die Verschärfungen der Bankenregulierung durch Basel III bleiben weit hinter den angekündigten Maßnahmen zurück. ${ }^{15}$ Die Ziele, die die EU mit den Regulierungen verfolgt, sind konsensuale internationale Entscheidungen, die sich an den USA orientieren. Sie blieben aber wegen der machtorientierten Strategien einiger Staaten bislang ohne großen Erfolg. ${ }^{16}$

Die Veränderungen der Finanzmarktregeln sollen hier allerdings nicht die zentrale Rolle spielen, da diese vorwiegend auf einer supranationalen Ebene zu verorten sind und dort andere Interessenkonstellationen zu finden sind als auf nationalstaatlicher Ebene. Wegen der schwierigen Ausgangslage für internationale Übereinkünfte haben Regierungen, so auch die deutsche Bundesregierung, frühzeitig begonnen, Reformen in Aussicht zu stellen, die innerhalb ihrer direkten Einflusssphäre liegen. Darunter fallen symbolische Regulierungsmaßnahmen wie die Begrenzung von Bonuszahlungen für Bankmanager und Reformen der institutionellen Finanzmarktaufsicht.

Die deutsche Finanzmarktaufsicht besteht in erster Linie aus der Bundesanstalt für Finanzdienstleistungsaufsicht (BaFin) - als weisungsgebundener Behörde im Geschäftsbereich des Finanzministeriums - und der Deutschen Bundesbank. Während die BaFin für die Aufsicht über Versicherungen und den Wertpapierbereich allein zuständig ist, teilt sie sich entsprechend dem Kreditwesengesetz (\$7 KWG) die Aufsicht über Banken mit der Deutschen Bundesbank, in deren Kompetenz die VorOrt-Prüfungen liegen. Insbesondere die Bankenaufsicht wurde nach der Finanzkrise zum Hauptgegenstand der Forderungen nach Reformen in der deutschen Finanzmarktpolitik. Weshalb blieben diese Reformen dennoch aus?

12 Basel II ist ein System der Regulierung von Bankgeschäften, das vom Basler Ausschuss für Bankenaufsicht bei der Bank für Internationalen Zahlungsausgleich erarbeitet wurde und sich vor allem auf die Festlegung bestimmter Eigenkapitalvorschriften stützt. Für den Versicherungsbereich konstruiert die Europäische Union in einer Art Komitologieverfahren, dem Lamfalussy-Verfahren, das Regelwerk Solvency II nach dem Vorbild von Basel II.

13 Vgl. Posner, Véron 2010, S. 400.

14 Clausen 2010.

15 Vgl. FTD vom 28.07.2010, »Neue Bilanzregeln kommen in entschärfter Version «.

16 Vgl. Posner, Véron 2010, S. 406. 
Das soll hier in drei Schritten nachgezeichnet werden. Zunächst werden drei Reformmodelle der Finanzmarktaufsicht erläutert, die seit 2009 die politische Debatte bestimmt haben. Die Reformdiskussion wird aus einer institutionalistischen Perspektive betrachtet, die Ergebnisse in Abhängigkeit von Akteurskonstellationen und strategischen, also instrumentellen und nutzenmaximierenden Verhaltensweisen erklärt. ${ }^{17}$ Institutionen umfassen dabei alle formalen und informalen Regeln, innerhalb derer sich Akteure bewegen, wobei im weiteren Verlauf vor allem formale Strukturen Berücksichtigung finden. ${ }^{18}$

Im zweiten Abschnitt werden die Akteursgruppen des Politikfelds Finanzen dargestellt. Dazu zählen neben den Marktteilnehmern selbst, also Finanzunternehmen und Verbrauchern, vor allem politische Akteure in Parteien, Parlament und Bundesregierung sowie die Aufsichtsbehörden des Finanzsektors. Schließlich folgt die Skizzierung der Interessen und Zielvorstellungen von politischen Akteuren, Marktteilnehmern und Aufsichtsbehörden. Aus einer Public Choice-Perspektive, die sowohl Kategorien der individuellen Nutzenmaximierung als auch der Gemeinwohlorientierung einschließt, werden dominante Strategien dieser Akteure herausgearbeitet, die verdeutlichen, welche grundsätzlichen Interessen mit Blick auf Regulierung und Aufsicht im Finanzmarkt bestehen. Im Fazit wird prognostiziert, dass tiefgreifende institutionelle Veränderungen in einer Zeit der Ruhe nach dem Sturm unwahrscheinlich sind.

\section{Politische Reaktionen auf die Krise}

Auf dem Höhepunkt der Finanzmarktkrise versuchte die deutsche Bundesregierung mit einer staatlichen Garantie für alle privaten Spareinlagen das gefährdete Vertrauen der Sparer wiederherzustellen. ${ }^{19}$ Diese Maßnahme sollte befürchtete Krisenfolgen wie Kapitalflucht und Kontenräumung verhindern, ebenso wie die Konjunkturpakete und die erweiterten Möglichkeiten zur Kurzarbeit gegen weitere befürchtete Krisenfolgen eingesetzt wurden. Gegen die Auslöser der Krise, insbesondere gegen fehlende oder unzureichende Risikoabsicherungen der Kreditinstitute, wurden höhere Mindestkapitalvorgaben, die Einführung einer Finanztransaktionssteuer und bessere Kundenberatungen vorgeschlagen. ${ }^{20}$ Mit dem sogenannten Restrukturierungsgesetz wurden neue Insolvenzregeln für Banken erlassen und ein Restrukturierungsfonds eingerichtet, der sich aus einer neu eingeführten Bankenabgabe speist und der Rettung von insolvenzbedrohten Banken dienen soll. Weitere Regulierungsmaßnahmen wurden im Baseler Ausschuss für Bankenaufsicht, in der European Insurance and Occupational Pensions Authority (EIOPA) und in anderen internationalen Gremien ergriffen, während sich die deutsche Bundesregierung auf eine Reform der nationalen Finanzmarktaufsicht konzentrierte.

17 Vgl. Hall, Taylor 1996.

18 Vgl. Peters 2005, S. 48.

19 Vgl. Manager Magazin 2008.

20 Vgl. BMF 2010.

Leviathan, 40. Jg., 1/2012 
Bereits im Koalitionsvertrag der Bundesregierung aus CDU, CSU und FDP wurde vereinbart, die Bankenaufsicht bei der Deutschen Bundesbank zu konzentrieren und der BaFin zu entziehen. ${ }^{21}$ Damit hatte ein Aufsichtssystem verändert werden sollen, das sieben Jahre zuvor von der Rot-Grünen Regierung gegen den Willen der Union eingeführt worden war. ${ }^{22}$ Die Festlegung auf die Konzentration der Bankenaufsicht bei der Bundesbank wurde zwar in Ansätzen durch wissenschaftliche Gutachten gestützt, war aber mit keinerlei organisatorischer Detailregelung oder auch nur der Operationalisierung des Ziels der Effizienzsteigerung verbunden. ${ }^{23}$ Die Zusammenlegung sollte Kommunikationsdefizite zwischen BaFin und Bundesbank beheben, die allerdings in keinem krisenrelevanten Fall belegt sind. ${ }^{24}$ Die Absichten der Bundesregierung blieben in ihrer finanzmarktpolitischen Wirkung symbolischer Natur und verfolgten eher parteipolitisch motivierte Ziele, insbesondere eine Maßregelung der BaFin. 25

Die inhaltlichen Lücken in den Plänen der Bundesregierung nutzten schließlich andere Akteure für Vorschläge zur Reform der Finanzaufsicht. Große Beachtung fanden das sogenannte »Integrationsmodell « der Deutschen Bundesbank und das »Holding-Modell« des Finanzpolitischen Sprechers der CDU-Bundestagsfraktion, Leo Dautzenberg.

Das von der Bundesbank favorisierte Integrationsmodell sah vor, die Aufsicht über alle Finanzbereiche vollständig der Bundesbank zu übertragen. ${ }^{26}$ Dies wäre einer Auflösung der BaFin gleichgekommen. Das Interesse der Bundesbank an einer solchen Lösung ist aufgrund des Aufgabenverlustes seit der Einführung des Euro begreiflich. ${ }^{27}$ Nicht abgebaute personelle Überkapazitäten erzeugen einen gewissen Rechtfertigungsdruck, dem die Bundesbank sich durch die Selbstzuschreibung neuer Aufgabenbereiche zu entziehen sucht. Mit dem Vorstoß, die BaFin vollständig in die Bundesbank einzugliedern, nutzte die Zentralbank die Gelegenheit, ein ihren tatsächlichen Kapazitäten angemessenes Modell auf die politische Agenda zu bringen. Gleichzeitig sollte mit der Integration der BaFin jedoch nicht auch die Ausdehnung der ministeriellen Rechts- und Fachaufsicht (vgl. $\$ 2$ FinDAG) auf die Bundesbank einhergehen. ${ }^{28}$

21 Vgl. Koalitionsvertrag 2009, S. 74.

22 Vgl. Frach 2008.

23 Vgl. Hüther et al. 2009; Wissenschaftlicher Beirat beim BMWi 2010.

24 Vgl. Hartmann-Wendels 2011; Interviewnachweis 1. Der Autor führte im Juli 2010 Interviews unter anderem mit Mitgliedern des Finanzausschusses im Deutschen Bundestag, einem ehemaligen hochrangigen Mitarbeiter der BaFin und der Behördenleitung der BaFin. In allen Fällen war eine anonyme Behandlung der Aussagen die Voraussetzung für die Interviewteilnahme, sodass die hier herangezogenen Interviews nur nummeriert angegeben werden können.

25 Vgl. Döhler 2011.

26 FTD vom 10.04.2010, „Streit um Finanzaufsicht. Bundesbank will das letzte Wort haben «.

27 Vgl. Frach 2008.

28 Vgl. Handelsblatt vom 29.11.2010,»Rolle rückwärts bei Finanzaufsicht«. 
Mit dem Holding-Modell sollten alle Zuständigkeiten der BaFin auf die Bundesbank verlagert werden. Der Plan der CDU sah die Schaffung einer dreisäuligen Bundesbankholding vor, in der die BaFin als eine Säule mit der vollständigen Aufsichtskompetenz über Banken, Versicherungen und Wertpapierhandel unter dem Dach der Bundesbank erhalten bliebe. ${ }^{29}$ Die weiteren Säulen sollten der Sonderfonds Finanzmarktstabilisierung (SoFFin) und die Bundesbank als Notenbank ohne Aufgaben in der Bankenaufsicht bilden. Da die BaFin-Aufsicht jedoch weiterhin unter der Fachaufsicht des BMF stehen sollte, wehrte sich die Bundesbank gegen dieses Modell, und auch die FDP übte Kritik, da sie die Unabhängigkeit der Zentralbank in Gefahr sah. ${ }^{30}$

Beide Modelle haben gemeinsam, dass sie Effizienzgewinne dadurch anstreben, dass die Aufsicht über Banken nicht mehr von zwei, sondern von einer einzigen Behörde wahrgenommen wird. Dabei wird das Ziel der "Effizienzsteigerung « jedoch in keiner Weise operationalisiert und verbleibt damit auf einer sehr abstrakten Ebene, die von einer reinen Kostenreduktion bis zu einer Totalkontrolle von Bankgeschäften reichen kann. Die Vorteile, die eine Konzentration der Bankenaufsicht und der Aufsicht über Versicherungen und Wertpapierhandel bei der Bundesbank hätte, sind schwer zu erkennen und lassen sich nicht aus objektiven Kriterien ableiten. ${ }^{31}$ Die Gründe, die es 2002 für die Schaffung einer Allfinanzaufsichtsbehörde gab - insbesondere die Herausforderung der Aufsicht über Finanzkonglomerate ,$-{ }^{32}$ sind nicht verschwunden, und auch das System der Allfinanzaufsicht stand nach der Krise nicht in Frage.

Trotz des Ausbleibens einer Reform der Finanzaufsicht stellt sich die Frage, weshalb es überhaupt zu der Diskussion um die Kompetenzverteilung zwischen BaFin und Bundesbank kam. Sah man die Reform als angemessene Lösung für aufgetretene Aufsichtsprobleme? Hierfür lassen sich keine empirischen Anhaltspunkte finden. Oder handelte es sich um das Ergebnis eines Problemlösungsprozesses, der unter Bedingungen beschränkter Rationalität stattfand? ${ }^{33}$ Solche Bedingungen treten häufig dann auf, wenn in einem Politikfeld schwerwiegende unerwartete Ereignisse eintreten und unter öffentlichem Druck und in kurzer Zeit scheinbar passende Entscheidungen getroffen werden, ${ }^{34}$ die sich dann jedoch als vorläufig erweisen. Die Reformmodelle für die deutsche Finanzmarktaufsicht lassen sich durchaus als Reaktionen auf die Finanzmarktkrise deuten, die keiner kausalen Logik folgen, in der also auf eine Problemanalyse keine angemessene Lösung folgt. Dennoch ist der Plan, der BaFin die Kompetenz für die Bankenaufsicht zu entziehen, nicht irrational und willkürlich gefasst worden, sondern fußt auf den strategischen Interessen einzelner

29 Vgl. Wallstreet online 2010.

30 Vgl. FTD vom 10.04.2010, »Streit um Finanzaufsicht. Bundesbank will das letzte Wort haben $«$.

31 Vgl. Greive, Jost 2009.

32 Vgl. Frach 2008.

33 Vgl. Cohen et al. 1972.

34 Vgl. Zahariadis 2007.

Leviathan, 40. Jg., 1/2012 
Akteure im Politikfeld. ${ }^{35}$ In den folgenden Abschnitten wird nicht nur das Politikfeld eingehender betrachtet, sondern auch die Behauptung geprüft, dass partikulare Interessen bezüglich der Steuerung von Behörden zu einer Entscheidungsblockade bei der Aufsichtsreform führten, die sich aus dem strategisch-instrumentellen Verhalten von Bundesbank, Bankenverbänden, politischen Parteien und anderen Akteuren ergab und letztlich institutionelle Veränderungen verhinderte.

\section{Politikfeld Finanzmarktpolitik}

An der Regulierung und Aufsicht in der Finanzmarktpolitik sind Akteure beteiligt, die sich einer der drei folgenden Gruppen zuordnen lassen: 1) Regulierer und Aufsicht, 2) Regelungsunterworfene und 3) regulierungsbegünstigte Dritte. Die letzte Gruppe, die Kunden der Finanzdienstleister, also Sparer und Investoren, spielt bei den Aushandlungsprozessen im Politikfeld keine direkte Rolle, wird aber von der Politik gewissermaßen treuhänderisch vertreten. ${ }^{36}$ Im Fall der Bundesrepublik Deutschland stehen auf der Reguliererseite neben den politischen Akteuren, der Bundesregierung und dem Deutschen Bundestag, in der Praxis die Aufsichtsbehörden, die BaFin und die Deutsche Bundesbank. Die Bundesbank ist eine weisungsfreie Behörde, ${ }^{37}$ die nach dem Kreditwesengesetz ( $\$ 7 \mathrm{KWG}$ ) für die Bankenprüfung vor Ort zuständig ist und der BaFin Berichte der Prüfungen zuleitet. Die BaFin wiederum ist eine weisungsgebundene Behörde, die nach dem Finanzdienstleistungsaufsichtsgesetz zum einen der Rechts- und Fachaufsicht des Bundesministeriums der Finanzen (BMF) unterliegt ( $\$ 2$ FinDAG) und zum anderen von einem Verwaltungsrat kontrolliert wird, der sich aus Vertretern von Bundesministerien, Bundestagsabgeordneten und Interessengruppenvertretern zusammensetzt (vgl. \$ 7 FinDAG). Trotz der ministeriellen Fachaufsicht genießt die BaFin eine gewisse institutionelle und budgetäre Unabhängigkeit. ${ }^{38}$ Bei der Erarbeitung von regulatorischen Vorschriften verhandelt die BaFin auf internationaler und europäischer Ebene als deutsche Vertreterin und kann dabei vor allem bei technischer Standardsetzung eigene Strategien verfolgen. ${ }^{39}$ Bei der Wahrnehmung der Aufsicht hat sie nicht nur ein weites Vollzugsermessen, sondern wirkt auf europäischer Ebene auch selbst an Implementierungsvorgaben für Regulierungen mit. Die relative Unabhängigkeit in der Aufsichtsund Regulierungsarbeit der BaFin ergibt sich aus der institutionellen Form, ihrem Status als Anstalt des öffentlichen Rechts, und durch die Budgetierung in Form der Umlagefinanzierung durch die Finanzinstitute. ${ }^{40}$

35 Vgl. Mügge 2010; Interviewnachweis 2.

36 Vgl. Handke 2010 b.

37 Vgl. Art. 88 GG; $\mathbb{} 12$ BundesbankG.

38 Vgl. Quintyn, Taylor 2004, S. 4.

39 Vgl. Handke 2010 b, S. 126.

40 Vgl. Handke 2010 b, S. 122. 
Die Unabhängigkeit der BaFin hat ihre Grenzen in der formal nahezu unumschränkten ministeriellen Fachaufsicht. ${ }^{41}$ Dies kann nicht nur den Umbau oder die interne Arbeitsweise der Behörde betreffen, sondern auch die Aufsichtsarbeit, sodass »die Regierung sich ohne eigene Kosten über Maßnahmen der Aufsichtsbehörde hinwegsetzen oder sie aussetzen kann, wenn Banken in Turbulenzen geraten sind, sie vor der Schließung retten kann und dadurch riskiert, der Gesellschaft künftig höhere Kosten aufzuladen «. ${ }^{42}$

Die Regierung tritt hier vorwiegend durch das Bundesministerium der Finanzen in Erscheinung, das unter anderem zuständig für Fragen der Haushaltspolitik, Währungspolitik, Finanzmarktregulierung und Finanzmarktaufsicht ist. Das BMF verfügt über ein spezielles Aufsichtsreferat für die BaFin. Dieses Referat VII A 7 steuert die nachgeordnete Behörde des BMF ausschließlich in den Bereichen Organisation, Haushalt und Personal (Interviewnachweis 3). Diese Steuerung geschieht "zielorientiert, ... vertrauensvoll [und mit dem Belassen der Erfüllung von] Aufgaben in eigener Zuständigkeit «. ${ }^{43}$ Dem vorgesetzten Ministerium steht ein Bündel von Maßnahmen zur Verfügung, um das rechtmäßige und zweckmäßige Verwaltungshandeln der BaFin sicherzustellen, wobei Berichts- und Informationspflichten zu den wichtigsten Elementen zählen. Je stärker ein Ministerium nicht nur nachträgliche Kontrolle ausübt, sondern auch im Vorfeld steuernd eingreift, desto stärker spielt »die Arbeitsbelastung der aufsichtsführenden Referate eine Rolle " als limitierender Faktor der Fachaufsicht. ${ }^{44}$ Es kommt damit zu einem Zielkonflikt zwischen der intendierten Autonomie der Behörde und dem nach wie vor vorhandenen Interesse des Ministeriums, bei Bedarf kontrollierend und steuernd tätig werden zu können. ${ }^{45}$

Dieses Steuerungsinteresse hängt wesentlich nicht zuletzt von den Parteien ab, die die Regierung bilden, sodass bei einer genaueren Betrachtung des Politikfelds im Zusammenhang mit der Finanzkrise die politischen Akteure noch weiter zu differenzieren wären. Da durch den Regierungswechsel 2009 auch die politikfeldspezifischen Präferenzen der Parteien Auswirkungen auf die Veränderungsvorstellungen bei der Aufsicht haben, werden diese als eigenständige Akteure mit grundlegenden Strategien der Staatsaktivität im Finanzmarkt behandelt.

Dies führt schließlich zu den Finanzunternehmen als den regulierten Akteuren, die sich als Einzelunternehmen oder als organisierte Interessenverbände im Politikfeld einbringen. Die Banken-, Versicherungs- und Wertpapierbranchen verfolgen jeweils eigene Geschäftsmodelle, die sich besonders hinsichtlich ihres Risikos unterscheiden. Aus diesem Grund gibt es kein homogenes Interesse der Finanzunternehmen, sondern nur ein je branchenspezifisches. Die Aufsicht trug diesem Unterschied in der traditionellen Trennung der ehemaligen deutschen Aufsichtsämter und aktuell

41 Vgl. Döhler 2002.

42 Vgl. Quintyn, Taylor 2004, S. 7.

43 BMI 2008, S. 2.

44 Ebd., S. 5.

45 Vgl. Aulich et al. 2010, S. 214.

Leviathan, 40. Jg., 1/2012 
in der Separierung der Aufsichtsbereiche innerhalb der BaFin Rechnung. In der Regulierung findet jedoch eine zunehmende Vereinheitlichung statt, da mit "Solvency II «, dem Regulierungspaket für Versicherungen, eine Kopie von Basel II, dem Regulierungspaket für Banken, konstruiert wird.

Ein bestimmendes Element der Finanzmarktpolitik, in dem sich Akteursinteressen gegenüberstehen, ist die staatliche Regulierung des Geschäftsbetriebs von Banken, Versicherungen und Wertpapierhändlern. Wie für andere Politikbereiche kann auch hier zunächst die Unterscheidung in Markt- und Risikoregulierung vorgenommen werden, um zu zeigen, welche Faktoren regulierungsbedürftig sind. ${ }^{46}$ Marktregulierung umfasst alle staatlichen Maßnahmen, die darauf gerichtet sind, einen funktionierenden Wettbewerb herzustellen oder aufrechtzuerhalten, zum Beispiel illegale Kartelle und Monopole zu entdecken und zu verbieten. In den Bereich der Risikoregulierung fallen hingegen Maßnahmen, die Verbraucher vor schädigenden Auswirkungen der Produktion oder des Vertriebs von Produkten und Dienstleistungen schützen. Zur Risikoregulierung zählen unter anderem Arzneizulassungsbestimmungen, Vorgaben für Produktstandards oder Hygiene- und Fütterungsstandards in der Nutztierhaltung.

Die deutsche Finanzmarktpolitik ist überwiegend ein Politikfeld für Maßnahmen der Risikoregulierung, mit denen das öffentliche Gut der Systemstabilität und -integrität geschützt werden soll. ${ }^{47}$ Der Schutz von Verbrauchern ist eine bedeutende Herausforderung für staatliches Handeln, da sich der Finanzmarkt ähnlich wie andere Bereiche, in denen Auftragsbeziehungen bestehen, durch ausgeprägte Informationsasymmetrien auszeichnet, die mehrheitlich zulasten der Verbraucher gehen. ${ }^{48}$ Diesen ist in der Regel nicht bekannt, in welcher geschäftlichen Situation sich eine Bank oder eine Versicherung befindet, bei denen sie Geld anlegen oder Risiken absichern. Selbst wenn die Informationen darüber grundsätzlich existieren, fehlt Verbrauchern häufig das Wissen, um diese zu verarbeiten..$^{49}$ Das Problem der »hidden information " soll durch staatliche Regulierung so gelöst werden, dass mündige Verbraucher ${ }^{50}$ in die Lage versetzt werden, Finanzverträge und deren Risiko selbst ausreichend beurteilen und eine entsprechende Vertragsentscheidung treffen zu können. In Fällen, in denen Verbraucher solche Beurteilungen nicht vornehmen können, soll die staatliche Aufsicht diese Aufgabe erfüllen und damit einerseits ersatzweise eine Vertrauensbasis für das Funktionieren des Marktes schaffen, andererseits Verbraucher vor bestimmten Risiken schützen. Neben diesem Verbraucherschutz verfolgt die Risikoregulierung den Schutz des Finanzsystems in seiner Gesamtheit, also für Verbraucher, Finanzunternehmen und indirekt betroffene Dritte.

46 Döhler 2006, S. 208.

47 Vgl. Handke 2010 a.

48 Vgl. Barth et al. 2004.

49 Vgl. Kessler 2008, S. 19.

50 Das Leitbild des »mündigen Verbrauchers « findet sich in verschiedenen Politikfeldern, so zum Beispiel im Lebensmittelbereich, im Gesundheitssektor oder auch in der Finanzmarktpolitik. 
Während ein kollektives Interesse an Finanzmarktstabilität besteht, gibt es hinsichtlich Art und Umfang der Risikoregulierung unterschiedliche Präferenzen. Es ist nicht überraschend, dass die individuellen Präferenzordnungen nicht ohne weiteres zu einer kollektiven Präferenzordnung aggregiert werden können, deshalb ist die Ausgestaltung des Systems der Finanzaufsicht kaum je konfliktfrei. ${ }^{51}$

\section{Akteursinteressen und Steuerungskonflikte}

Es gibt historische Schlüsselereignisse, die die institutionelle Ordnung innerhalb eines Politikfelds grundsätzlich aufbrechen und verändern können. Ein massiver externer Schock muss jedoch nicht zwangsläufig bedeuten, dass »bewährte Regelsysteme ... einfach ihre frühere Leistungsfähigkeit einbüßen und deshalb unmittelbar nach politischer Reform verlangen.$^{52}$ Vielmehr bleibt auch in der Finanzkrise die Veränderung der Regulierungen abhängig von internationalen Machtkonstellationen, ebenso wie Aufsichtsreformen von nationalen Konstellationen determiniert werden. Akteure in der Finanzmarktpolitik streben nach je eigenen Zielen, wobei Präferenzen hinsichtlich Regulierung und Aufsicht an Strategien zur Nutzenmaximierung ausgerichtet sind. Davon betroffen ist auch die Finanzaufsicht nach der Krise, wobei allein der Kairos, also der passende historische Augenblick (oder das » window of opportunity «), keine Garantie für tatsächlichen Politikwandel ist. Der Grund hierfür ist, dass »[v]eränderte Problemlagen ... von den an Veränderung interessierten Akteuren als Gelegenheit wahrgenommen [werden], ihre politische Vorstellung einer veränderten Nutzung oder Neuordnung von Institutionen durchzusetzen, während die am Status quo interessierten Kräfte jede Neuordnung verhindern wollen ", wie Roland Czada am Beispiel der Arbeitsmarkt- und Sozialpolitik erklärt. ${ }^{53}$ Der Wandel von Institutionen hängt also von der Konstellation und vom Verhalten von Akteuren ab, die definierbare Präferenzen haben, an denen sie festhalten. In ihre Strategie der Nutzenmaximierung beziehen sie auch Einschätzungen über das erwartete Verhalten anderer Akteure in die eigenen Aktivitäten mit ein.

Die Akteure in der Finanzmarktpolitik setzen sehr unterschiedliche Prioritäten hinsichtlich Regulierung, Aufsicht und eigener Beteiligung an der Politikformulierung und -implementation. Das BMF, die BaFin und die Bundesbank sind bürokratische Organisationen, für die angenommen wird, dass sie einerseits regelkonform ihre zugewiesenen Aufgaben erfüllen, andererseits aber auch organisationsspezifische Eigeninteressen verfolgen, nämlich in erster Linie den Wunsch nach Fortbestand und Ausbau der Behörde sowie die Maximierung des Budgets. ${ }^{54}$ Aus diesem Interesse resultiert die Strategie von Behörden, ihren Aufgabenbereich als überaus wichtig und ressourcenintensiv - eventuell auch stärker als für die Aufgabenerfüllung nötig - darzustellen, um mehr finanzielle und personelle Mittel zu er-

51 Vgl. Arrow 1963.

52 Czada 2003, S. 17.

53 Ebd.

54 Vgl. Niskanen 1979.

Leviathan, 40. Jg., 1/2012 
halten. Diese Mittel werden - so eine neidvolle Interpretation des Behördenhandelns - für individuelle »Annehmlichkeiten« der Mitarbeiter verwendet. In der Regel werden Ressourcen der Organisation jedoch zur Erreichung von programmatischen Zielen und zur Festigung der Behördenposition im Politikfeld verwendet. ${ }^{55} \mathrm{Zu}$ dieser Verankerung im Politikfeld gehört auch, dass sich eine Behörde und ihre Mitarbeiter möglichst aus öffentlich ausgetragenen Konflikten heraushalten, um nicht Gegenstand politischer oder medialer Kritik zu werden. Dieses Verhalten ist an das sogenannte »bureau shaping " angelehnt, das auf eine möglichst angenehme und konfliktarme Aufgabenerfüllung innerhalb der Behördenarbeit abzielt. ${ }^{56}$ Die drei beschriebenen Strategien des rationalen Verhaltens in und von bürokratischen Organisationen sind die Grundlage für die folgende Betrachtung der Handlungsmuster von BMF, BaFin und Bundesbank.

\section{$B M F$}

Das deutsche Finanzministerium verfügt mit der Bundesfinanzverwaltung über einen eigenen Verwaltungsunterbau bis auf die Ebene örtlicher Behörden. Darüber hinaus befinden sich acht weitere nachgeordnete Behörden im Geschäftsbereich des Ministeriums, zu denen die BaFin als größte eigenständige Behörde gehört. ${ }^{57}$

Durch ihre Konstruktion als Anstalt des öffentlichen Rechts und die Finanzierung durch ein Gebührensystem und andere Elemente, die durch das Finanzdienstleistungsaufsichtsgesetz FinDAG festgelegt sind, ist die BaFin dem Behördentypus der unabhängigen Agentur sehr nah. ${ }^{58}$ Die Zugriffsmöglichkeiten des Ministeriums sind somit durch eine gewisse politisch intendierte Selbstständigkeit der BaFin begrenzt. Gleichzeitig unterliegt die Behörde der formal unbeschränkten Weisungskompetenz des BMF, wodurch ein Konflikt institutionalisiert ist, der in der deutschen Behördenlandschaft einmalig ist. Gründe für die Einrichtung einer relativ unabhängigen Behörde waren einerseits, auf diese Weise den Anschein reiner, objektiver Fachlichkeit für die Wahrnehmung der Aufsicht zu erwecken, andererseits aber auch eine Institution im Politikfeld zu etablieren, die unbequeme Entscheidungen treffen kann, ohne dass Kritik daran direkt auf das Ministerium zurückfällt. Dieses Verschieben der Verantwortung (»blame shifting «) ist als wesentlicher Grund für die Errichtung unabhängiger oder quasi-unabhängiger Agenturen zu erachten und besitzt aus der Nutzenüberlegung des BMF auch für die BaFin Gültigkeit. ${ }^{59}$ Gleichzeitig ist das BMF daran interessiert, dass die BaFin ihre Aufgaben so erfüllt, dass möglichst wenige Konflikte und Störungen auftreten und keine Problemlösungszwänge für das Ministerium entstehen. Aus Sicht eines Ministeriums mit begrenzten Ressourcen und Informationsverarbeitungskapazitäten ist die Arbeit einer nachgeordneten Behörde ideal, wenn sie wenig eigene Ressourcen bindet und sich die

55 Vgl. Downs 1967, S. 88 f.

56 Vgl. Dunleavy 1991.

57 Vgl. BMF 2011.

$58 \mathrm{Vgl}$. Aulich et al. 2010.

59 Vgl. Ebinger, Schmitt 2010. 
Fachaufsicht ohne größeren Zeitaufwand ausüben lässt. ${ }^{60}$ Dies bedeutet, dass die Wahrnehmung der Fachaufsicht sich auf allgemeine Kontrolle und fallweise Steuerung beschränken kann und direkte Eingriffe per Weisung nur in Ausnahmefällen vorkommen. ${ }^{61} \mathrm{Im}$ Fall der BaFin zeigte sich jedoch, dass das BMF die Möglichkeit, regulatorische Ziele zu definieren und Aufsichtsmaßnahmen durch Anweisung zu bestimmen, in Gefahr sah. Das eigenständige Agieren der BaFin während der Basel II-Verhandlungen, die Einbindung in europäische Gremien und ein sehr selbstbewusstes Auftreten der Behördenspitze führten dazu, dass das BMF einschritt, um die BaFin in ihrer Emanzipationsentwicklung zu beschränken. ${ }^{62}$ Die Leitungsstruktur wurde von einer Präsidial- zu einer Kollegialstruktur umgebaut, und Richtlinien zur Wahrnehmung der ministeriellen Rechts- und Fachaufsicht über die Behörde wurden detailliert fixiert und auf der Internetseite der BaFin veröffentlicht. Mit der Präzisierung der Fachaufsicht entsteht für die BaFin eine Bringschuld gegenüber dem Ministerium, die jeden Bereich ihrer aufsichtlichen und regelschaffenden Arbeit umfasst. Das BMF, das »die Umsetzung der Richtlinien der Bundesregierung nicht im Einzelfall überprüft ", 63 ist mit den allgemeinen und pauschalisierten Konkretisierungen der Fachaufsicht seinem Eigeninteresse nach einem möglichst geringen Ressourceneinsatz für den nachgeordneten Bereich gefolgt. Der Verpflichtung, das BMF frühzeitig über Erkenntnisse und Schritte der Aufsicht zu informieren, ist die BaFin im Vorfeld und während der Finanzkrise nachgekommen. Am Beispiel der Bank Hypo Real Estate (HRE) zeigte sich jedoch, dass die geringe Aufsichtsintensität, die im Regelfall »nur das Abheften und Ablegen von Meldungen der BaFin durch Mitarbeiter des mittleren Dienstes « ${ }^{64}$ umfasste, unzureichend war. Nicht nur eine ausreichende Steuerung und Kontrolle der BaFin ist dem BMF unmöglich, sondern auch das aktive Handeln im Politikfeld selbst. Aufgrund der unzureichenden Personalausstattung ${ }^{65}$ und der fehlenden Spezialkenntnisse der Mitarbeiter des Ministeriums war dieses auf dem Höhepunkt der Krise nicht in der Lage, wichtige Gesetze, etwa das Gesetz zur Ergänzung des Kreditwesengesetzes, selbst zu erarbeiten und musste diese Aufgabe an Rechtsanwaltskanzleien auslagern. ${ }^{66}$

Mit der Abteilung VII und vor allem dem dort angesiedelten Aufsichtsreferat VII A7 ist das Ministerium nicht nur bemüht, die nachgeordnete Aufsichtsbehörde hinsichtlich der Regulierung an die Präferenzen des Hauses zu binden, sondern auch

60 Vgl. Bundesrechnungshof 2005, S. 17.

61 Vgl. Döhler 2007, S. $238 \mathrm{ff}$.

62 Vgl. Sturm et al. 2002, S. 36; Interviewnachweis 4.

63 Bundestags-Drucksache 16/3253 zur Rolle der Bundesregierung bei der Rechts- und Fachaufsicht der Bundesanstalt für Finanzdienstleistungsaufsicht (BaFin), S. 4.

64 Interviewnachweis 5.

65 Der Personalbestand der Abteilung VII ist, entsprechend einer Antwort des Ministeriums auf eine Anfrage nach dem Informationsfreiheitsgesetz durch den Autor, auf niedrigem Niveau stabil. Die Abteilung verfügt über 120 Mitarbeiterinnen und Mitarbeiter, die auf 19 Referate verteilt sind.

66 Vgl. Hanke 2009; Interviewnachweis 6.

Leviathan, 40. Jg., 1/2012 
zu kontrollieren und zu steuern. Darin zeichnet sich ein Konflikt zwischen BMF und BaFin ab, der die Steuerung in Bezug auf regulatorische Zieldefinitionen ebenso umfasst wie das aufsichtliche Tagesgeschäft. Die BaFin ist an einer möglichst unabhängigen Aufgabenerfüllung nach fachlichen Maßstäben interessiert, während das BMF zwar ebenfalls die wirkungsvolle Umsetzung der regulatorischen Bestimmungen durch die Aufsicht anstrebt, gleichzeitig aber in der Lage sein möchte, die Behörde je nach Bedarf zur Aufnahme oder Aussetzung von aufsichtlichen Aktivitäten anzuweisen. ${ }^{67}$ Als Beispiele lassen sich die Basel II-Verhandlungen, in denen die Arbeit an einem internationalen Regulierungsregime von der BaFin allzu selbstständig wahrgenommen wurde, ${ }^{68}$ und die Aufsicht über einzelne Institute nennen, bei denen Prüfungen auf Weisung des BMF verhindert wurden. ${ }^{69}$ Gerade die Erteilung von Weisungen, die den Aufgabenbereich der Aufsicht in konkreten Einzelfällen betreffen, zeigt, dass das BMF von politischen Erwägungen und Einflüssen von organisierten Interessen nicht frei ist. Dies ist ein Grund dafür, weshalb einerseits das BMF selbst ein Interesse daran hat, die Steuerbarkeit der Behörde nicht zu gefährden, und andererseits auch Interessengruppen danach streben, über den Umweg des BMF auf die Arbeit der BaFin Einfluss zu nehmen. Das BMF steht bei der Steuerung der BaFin somit nicht nur in Konkurrenz zu den fachlichen Eigeninteressen der Aufsichtsbehörde, sondern auch zu den Interessen anderer Finanzunternehmen, die keinen direkten Kontakt zum Ministerium haben, sondern eine besondere Nähe zu der Behörde suchen. ${ }^{70}$ Dies bedeutet, dass "sowohl die regulierte Interessengruppe als auch der Gesetzgeber bzw. die Regierung versuchen, Anreizmechanismen zu implementieren, die geeignet sind, die Aktivitäten der Aufsichtsbehörde im gewünschten Sinne zu beeinflussen $« .{ }^{71}$ Die Positionierung der BaFin steht daher besonders im Fokus einer Nutzenuntersuchung im Akteursfeld.

\section{BaFin}

Die Allfinanzaufsicht BaFin hat zwei wesentliche Aufgaben im Finanzbereich: Einerseits ist sie - auf europäischer und internationaler Ebene - an der Konstruktion von Regulierungsregimen wie Basel II und Solvency II beteiligt, andererseits ist sie mit der Implementation dieser und anderer Vorschriften betraut ( $\$ 4$ Fin DAG). Die tatsächliche Beaufsichtigung von Finanzinstituten findet dabei ausschließlich auf nationaler Ebene statt. Dabei hat die BaFin gewisse Spielräume im Rahmen ihres gesetzlichen Aufsichtsermessens (vgl. KWG). Bei ihrer Aufgabenerfüllung befindet

67 Vgl. Interviewnachweis 7.

68 Vgl. Döhler 2007, S. 35.

69 Vgl. Interviewnachweis 8.

70 Durch die besondere Nähe zwischen Regulierungs- bzw. Aufsichtsbehörde und Regelungsunterworfenen besteht die Gefahr der Vereinnahmung einer Behörde durch den Regulierungsbereich und die dort wirkenden Gruppen. In einem solchen Fall besteht ein sogenanntes »agency capture (vgl. Stigler 1971), das jedoch im Fall der BaFin nicht nachzuweisen ist.

71 Kaserer 2006, S. 76. 
sie sich jedoch immer in einem Spannungsverhältnis zwischen wettbewerbspolitischen und sozialen Zielen, die darüber hinaus mit einer »Vielzahl widersprüchlicher Zielvorgaben « 72 von Seiten der Politik verknüpft sein können.

Bei der Aufsichtsarbeit kann sich die BaFin trotz aller Ermessensspielräume den Weisungen des BMF nicht entziehen, wenn dieses die Fachaufsicht wahrnimmt und dadurch direkt in Prüfungs- und Kontrollaufgaben eingreift. Anders stellt sich dies bei der Mitwirkung an Regulierungsvorschriften dar. Die BaFin unterliegt zwar auch hier der Fachaufsicht, kann sich aber, wenn mit dem BMF auf nationaler Ebene keine Einigung erzielt werden kann, auf ihre europäische oder internationale Kompetenz berufen. In Fällen, in denen Uneinigkeit zwischen BMF und BaFin besteht, ist es der Behörde also möglich, mit Verweis auf internationale oder europäische Gremien wie den Baseler Ausschuss oder das Committee of European Insurance and Occupational Pensions Supervisors (CEIOPS) zu agieren. Gerade CEIOPS war, wie auch die Schwesterausschüsse für Banken und Wertpapierhandel, durch seine Besetzung mit nationalen Experten sehr angesehen und hatte bei privaten und staatlichen Akteuren ein hohes Maß an Legitimität erreicht, obwohl im Rahmen des Lamfalussy-Verfahrens von diesem Ausschuss keine verbindlichen Entscheidungen getroffen wurden. ${ }^{73}$ Innerhalb der neuen Aufsichtsagenturen EBA, ESMA und EIOPA soll die BaFin sogar eine völlige Weisungsfreiheit von nationalen Regierungen genießen, wobei sich erst in der Zukunft zeigen wird, wie sich so etwas faktisch realisieren lässt. ${ }^{74}$ Für die BaFin scheint bislang allerdings zuzutreffen, dass "politisch brisante Entscheidungen ... nie nach rein fachlichen Kriterien gefällt [werden], unabhängig davon, wie autonom diese organisiert sind . $^{75}$ Dies betrifft einerseits Maßnahmen der Aufsicht, andererseits aber auch Bereiche der Behördenorganisation, zu denen BaFin-Mitarbeiter bestimmte eigennützige Positionen vertreten. Aufgrund dessen ist es für die BaFin eine rationale Strategie, Aufsicht und Regulierung als zentrale und unverzichtbare Elemente der Finanzmarktpolitik darzustellen und damit Argumente für zusätzliche Ressourcen zu liefern. ${ }^{76}$

Das Bundesaufsichtsamt für das Kreditwesen (BaKred) hatte es auf diese Weise zwischen 1988 und 2001 bereits geschafft, die Anzahl seiner Planstellen um 270 Prozent zu steigern, und auch die BaFin als seine Nachfolgeorganisation konnte den Mitarbeiterbestand von 2002 bis 2005 um 30 Prozent erhöhen. ${ }^{77}$ Die BaFin, der auch für das Jahr 2011 mehr als 240 zusätzliche Stellen bewilligt wurden, ist damit eine wachsende Behörde, die jedoch einen großen Teil ihrer personellen Ressourcen nicht direkt für die Aufsicht einsetzt, sondern für Querschnittsfunktionen und Aufgaben der internen Koordination. ${ }^{78}$

72 Eberlein 2000, S. 96.

73 Vgl. Posner, Véron 2010, S. 405.

74 Vgl. Commission of the European Communities 2009.

75 Ebinger, Schmitt 2010, S. 20.

76 Vgl. Sanio 2002.

77 Vgl. Kaserer 2006, S. 69, 73.

78 Vgl. BaFin 2010 a, 2010 b. 
Ein weiteres Bestreben der BaFin besteht darin, sich der Kontrolle des BMF zumindest insoweit zu entziehen, dass die Anmeldung eines hohen Aufsichtsbedarfs durch das Ministerium nicht infrage gestellt wird. Dieses Ziel kann durch eine starke fachliche Kompetenzbündelung der BaFin erreicht werden, die ihr einen Informationsvorsprung gegenüber dem BMF gibt. Zum Ausdruck kommt dieser Vorsprung aber auch bei der Erarbeitung von Regulierungsregimen, die wie Basel II und Solvency II einen so hohen technischen Komplexitätsgrad erreichen, dass das BMF sich auf die Expertise der BaFin verlassen muss. ${ }^{79}$ Die Folge ist der bereits angesprochene Konflikt zwischen BMF und BaFin, der entsteht, weil sich die BaFin von der faktischen Fachaufsicht des BMF entfernen kann, weil das BMF nicht die Möglichkeit hat, die Fachaufsicht bei Bedarf mit beliebiger Intensität ausüben zu können. ${ }^{80} \mathrm{Da}$ hinter verbirgt sich ein Delegationsproblem, das darin besteht, dass das BMF die BaFin nur eingeschränkt steuern und kontrollieren kann, da das Ministerium hierfür nicht genügend qualifiziertes Personal zur Verfügung hat. ${ }^{81}$

\section{Bundesbank}

Schon lange vor der jüngsten Finanzkrise spielte die Frage eine Rolle, welche Aufgabe Zentralbanken bei der Finanzmarktaufsicht übernehmen sollen. ${ }^{82}$ Die Bundesbank strebte eine alleinige Zuständigkeit für die Bankenaufsicht unter ihrem Dach an, konnte aber die Gründung der BaFin nicht verhindern. ${ }^{83}$ Die Übertragung der alleinigen Bankenaufsicht auf die Bundesbank hätte einen großen politischen Steuerungsverlust bedeutet, der nicht gewollt war. ${ }^{84}$ Mit der Abgabe von geldpolitischen Aufgaben an die EZB nach der Einführung des Euro haben sich bei der Bundesbank personelle Überkapazitäten ergeben, sodass sie trotz Einbußen bei ihren Betätigungsfeldern derzeit noch immer über etwa 9.000 Mitarbeiter verfügt, während die BaFin nur knapp 2.000 Beschäftigte hat. Es entspricht somit der Präferenz für die Aufrechterhaltung und den Ausbau einer Behörde, dass die Bundesbank die Notwendigkeit ihrer Zuständigkeit für die Bankenaufsicht herausstreicht. Insgesamt verfolgen Bundesbank und BaFin daher sehr ähnliche Ziele für ihre jeweilige Organisation. Mit dem Integrationsmodell brachte die Bundesbank in einer Art Präventivschlag ein Modell der Aufsicht in die politische Debatte ein, das alternative Vorschläge im Keim ersticken und den Führungsanspruch der Notenbank im Reformprozess untermauern sollte. ${ }^{85}$

Die Interessen von BaFin und Bundesbank gingen dadurch aber in verschiedene, jeweils eigennutzorientierte Richtungen, was Kompetenzen und Ressourcen anbelangte. Das Verhältnis beider Behörden ist in der Bankenaufsicht seit 2002 durch

79 Vgl. Interviewnachweis 9.

80 Vgl. Döhler 2011.

$81 \mathrm{Vgl}$. Interviewnachweis 10.

82 Vgl. Heinemann, Schüler 2004, S. 99.

83 Vgl. Frach 2008, S. 65.

84 Vgl. Döhler 2008, S. 269.

$85 \mathrm{Vgl}$. Interviewnachweis 11. 
eine horizontale Trennung der Aufsichtskompetenz gekennzeichnet, die zu einer Konkurrenzsituation führte, in der "Grabenkämpfe, Konflikte um Politikmaßnahmen oder Versuche, Schuldzuweisungen abzuwehren " ${ }^{86}$ die Regel sind. Um dabei erfolgreich zu sein, nutzten Bundesbank und BaFin während der Reformdebatte den Kontakt zu ihnen nahestehenden (partei-)politischen Akteuren. Parteien erweisen sich daher als weiterer wichtiger Faktor im Politikfeld.

\section{Parteien}

In der Public Choice-Theorie unterscheidet man bei der Politik zwei wesentliche Modelle des rationalen Verhaltens: zum einen die Rationalität in bürokratischen Organisationen, zum anderen die Rationalität von Politikern. ${ }^{87}$ Dabei wird das individuelle Verhalten von Politikern, die nach (Wieder-)Wahl, Macht, finanziellen Ressourcen, Prestige und politischen Zielen streben, auf Kollektivinteressen, das heißt auf Parteien, übertragen. ${ }^{88}$ Die Interessen von Parteien an der Ausgestaltung der Finanzaufsicht in Deutschland sind verschieden. So lässt sich beobachten, dass die SPD keine Notwendigkeit einer Auseinandersetzung mit Fragen der Finanzaufsicht sieht. ${ }^{89}$ Die Regierungsparteien hingegen befassen sich mit dieser Frage und verfolgen dabei Ziele, die zum Teil mit nutzenmaximierendem Verhalten erklärbar sind. Dazu wird hier die Konzeption zur Regierungstätigkeit von Andrei Shleifer und Robert Vishny verwendet. Mit deren modellhaften Beschreibungen politischen Handelns, invisible hand, grabbing hand und helping hand, wird im Folgenden Regierungsarbeit analytisch erfasst und bewertet. ${ }^{90}$ Die invisible hand ist eine präskriptive Konzeption, die sich an liberalistische Ideen anlehnt und dem Markt alle Aufgaben überlässt. Im Gegensatz zu den beiden anderen Modellen ist hier die politische Auseinandersetzung (politics) bedeutungslos, weshalb eine Untersuchung von Parteistrategien aus dieser Sicht überflüssig ist. ${ }^{91}$ Die helping hand-Perspektive, die ursprünglich ebenfalls einen präskriptiven Charakter hatte, führt an dieser Stelle weiter, da sie die soziale Wohlfahrt als übergeordnetes Ziel jeder Regierungstätigkeit begreift. Shleifer und Vishny konzentrieren sich jedoch auf das grabbing handVerhalten von Regierungspolitikern und -parteien, das einen Schwerpunkt auf politics legt und der Staatstätigkeit im Markt skeptisch gegenübersteht. ${ }^{92}$

Nicht nur nach der Finanzkrise, sondern auch schon einige Zeit davor gewannen "Aspekte des Konsumentenschutzes ein immer stärkeres Gewicht gegenüber Sys-

86 Hood et al. 2003, S. 127.

87 Vgl. Lane 1990, S. 18.

88 Vgl. ebd.

89 Die SPD ist seit Herbst 2009 in der Opposition und widmet sich in dieser Rolle den Bereichen der Finanzmarktregulierung und -aufsicht nur in geringem Umfang. Die Partei sieht vor allem in der Finanzmarktaufsicht keinen Handlungsbedarf und möchte den Status quo beibehalten (Interviewnachweis 3).

90 Vgl. Shleifer,Vishny 1999.

91 Vgl. ebd., S. 3.

92 Vgl. ebd.

Leviathan, 40. Jg., 1/2012 
temstabilisierungsargumenten in der Diskussion um die Aufsicht $« .{ }^{93}$ Dies bringt eine starke Gemeinwohlorientierung zum Ausdruck, die durch das helping hand-Modell erfasst wird und das Bemühen einer Regierung zum Ausdruck bringt, in einer Gesellschaft die soziale Wohlfahrt zu maximieren. Parteien wie die SPD, Bündnis90/ DIE GRÜNEN und DIE LINKE lassen sich diesem Lager zuordnen. Aus ihrer Sicht bestehen in allen Bereichen des Marktes Defizite, die Staatseingriffe nicht nur erklären, sondern auch präskriptiv rechtfertigen. ${ }^{94}$ Regierungen, die eine helping hand-Überzeugung vertreten, handeln bei der Konstruktion der Aufsicht so, dass »der Glaube an den Markt ersetzt wird durch ein Vertrauen in den öffentlichen Dienst und den Glauben daran, dass Regierungsprogramme mit geringeren Kosten gehandhabt werden können als Märkte «. ${ }^{95}$ Dies impliziert, dass eine starke Finanzaufsichtsbehörde erwünscht ist, die mit einer ausgeprägten Sachorientierung Regeln implementiert. So ist das Modell der Allfinanzaufsicht BaFin auch eine Konstruktion der Rot-Grünen Bundesregierung, die von diesen Parteien zudem aktuell nicht ernsthaft infrage gestellt wird. ${ }^{96}$ Im Kern geht es helping hand-Politikern um politisch-administrative Steuerung, die Marktdefizite wie negative Externalitäten, Monopolbildungen oder Informationsasymmetrien zu begrenzen sucht. Einerseits sollen Verbraucher also durch »Regeln, die Überwachung ihrer Einhaltung und die Sanktionierung von Verstößen" geschützt werden, andererseits sind diese Regeln die Voraussetzung dafür, dass »der Markt zu einem level playing field [wird, also einem Ort mit gleichen Wettbewerbsbedingungen für alle], auf dem allein Leistung über Gewinn oder Verlust entscheidet «. ${ }^{97}$

Die deskriptive grabbing hand-Konzeption schließlich unterstellt, dass Regierungen grundsätzlich eine Vielzahl von Zielen verfolgen, die nicht speziell gemeinwohlfördernd oder auf den Ausbau des Wohlfahrtsstaates gerichtet sind. Vielmehr lässt sich aus dem Modell ableiten, dass »Regierungen sich egoistisch verhalten, ...entweder zu ihren eigenen Gunsten oder zugunsten derer, die sie unterstützen ", ebenso wie sich auch Staatsbedienstete eigennutzorientiert verhalten. ${ }^{98} \mathrm{Im}$ Vertrauen auf Marktprozesse wird zudem eine Marktsteuerung des Verhaltens von Akteuren im Finanzmarkt angestrebt. Weder sollten Banken in staatlichem Besitz sein, noch sollten staatliche Institutionen in der Lage sein, Marktprozesse über Gebühr zu beeinflussen. Die grabbing hand-Strategie kommt daher zu der politischen Handlungsempfehlung, dass für die Fälle, in denen Regulierung und Aufsicht unvermeidbar sind, »Beamte bei ihren Entscheidungen so wenig Ermessensspielräume haben sollen wie möglich «.99 In der deutschen Parteienlandschaft lassen sich CDU, CSU und FDP der Gruppe von Parteien zuordnen, die in der Finanzmarktpolitik eher nach der

93 Kaserer 2006, S. 71.

94 Vgl. Shleifer, Vishny 1999, S. 11.

95 Andolfatto 2004, S. 5.

96 Vgl. Interviewnachweis 12.

97 Czada 2003, S. 18.

98 Andolfatto 2004, S. 6.

99 Vgl. Shleifer,Vishny 1999, S. 12. 
grabbing hand-Logik agieren und für ihre Klientel eine Aufsicht anstreben, die keine zu große Machtfülle innehat. ${ }^{100}$ Diese Zuordnung der Parteien gilt jedoch nicht unbedingt für andere Politikfelder.

Parteien können innerhalb eines gewissen Rahmens - unterhalb der Offensichtlichkeitsschwelle - Regulierung und Aufsicht so gestalten, dass sie den Präferenzen der ihnen nahestehenden Industrie entgegenkommen. Solange dieses Entgegenkommen unentdeckt bleibt, besteht für die politischen Akteure nicht die Gefahr, durch die Klientelpolitik Wählerstimmen aus dem Lager derer zu verlieren, die nicht begünstigt wurden. ${ }^{101}$ Zwar vertreten Hood et al. die Auffassung, dass Klientelpolitik bei Risikoregulierung nicht regelmäßig, sondern empirisch nur in wenigen Fällen vorkommt. ${ }^{102}$ Im Fall der deutschen Aufsicht über diese Risikoregulierung zeigt sich die Berücksichtigung von Klientelinteressen nach dem Regierungswechsel 2009 jedoch sehr deutlich. ${ }^{103}$

Im Hinblick auf die Unabhängigkeit und Selbstständigkeit der BaFin hat die Verfolgung einer helping hand-Strategie oder grabbing hand-Strategie entscheidende Auswirkungen. Ändert sich die Zusammensetzung einer Regierung, dann ändern sich häufig auch die Präferenzen bezüglich institutioneller Strukturen. Während eine Regierung mit helping hand-Prägung eher » eine stabile Balance zwischen politischer Kontrolle, politischer Verantwortlichkeit und notwendiger Autonomie und fachlichen Unabhängigkeit von Behörden « anstrebt, kommt es bei Regierungen der grabbing hand-Orientierung mit großer Wahrscheinlichkeit dazu, dass »diese Balance nicht gehalten wird «. ${ }^{104}$ Eine solche Präferenzänderung, wie sie hier zu erkennen ist, hängt in der Regel mit dem Auftauchen von Interessen der Marktakteure, das heißt der Finanzunternehmen und ihrer Verbände, zusammen.

\section{Finanzunternehmen und Interessengruppen}

Zur Wahrung ihrer Interessen sind Finanzunternehmen mit allen zentralen Akteuren in Kontakt, die Entscheidungen im Politikfeld treffen. Priorität haben dabei Kontakte zu politischen Parteien mit deckungsgleichen Interessen und zu Regierungsparteien. ${ }^{105}$ Die Branche nutzt alle verfügbaren Einflussmöglichkeiten, ist dabei »gut organisiert und verfügt über starke Instrumente, um mit Informationen zu operieren und legislative Prozesse, zum Beispiel durch Spenden, Wahlunterstützung und die Einspeisung von einseitigen Information, zu beeinflussen «. ${ }^{106}$ Gegenüber der Aufsicht haben Unternehmen einen Informationsvorsprung, der sie in die Lage

100 Vgl. Dalla Pellegrina, Masciandaro 2008, S. 292 f.

101 Vgl. Heinemann, Schüler 2004, S. 101.

102 Vgl. Hood et al. 2003, S. 117.

103 Vgl. Kaiser 2010.

104 Vgl. Aulich et al. 2010, S. 214.

105 Vgl. Beyers 2009, S. 20.

106 Heinemann, Schüler 2004, S. 101. 
versetzt, regulatorische Lücken zu nutzen und sich aufsichtlicher Kontrolle unter Umständen zu entziehen. ${ }^{107}$

Finanzunternehmen streben nach maximaler unternehmerischer Freiheit, haben aber gleichzeitig ein Interesse daran, dass ein »funktionierender staatlicher Rahmen [besteht, der] eine notwendige Voraussetzung für die Interessendurchsetzung einzelner ökonomischer Akteure « 108 ist. Sobald Institute als Regulierungsunterworfene auftreten, streben sie jedoch danach, dass die Finanzmarktregulierung möglichst schwach ausgelegt ist und mehr konkurrenzbeschränkende Marktregulierung gegenüber ausländischen Wettbewerbern enthält. Gerade der Finanzsektor ist jedoch in dieser Präferenz nicht homogen, weil Versicherungen und Banken in Form von Sparkassen, Genossenschaftsbanken und Privatbanken höchst unterschiedliche Geschäftsmodelle betreiben. ${ }^{109}$ Sie sind von Regulierung nicht in gleicher Weise betroffen, da deren Wirkung von den jeweiligen Geschäftsmodellen und der Größe des Marktes, auf dem Institute agieren, abhängt. ${ }^{110}$ Weitgehend homogene Präferenzen bestehen bei Banken aller Branchen und bei Versicherungen jedoch hinsichtlich der Aufsicht. Diese wird von allen Instituten gleichermaßen als belastender Kostenfaktor angesehen, den es zu minimieren gilt. Kosten entstehen zum Beispiel durch Dokumentationsaufgaben, bei der Erstellung von Berichten an die BaFin oder der Bereitstellung von Informationen und Räumen bei Vor-Ort-Prüfungen, sodass Finanzinstitute ein Interesse an lockerer, koordinierter und nicht zu häufiger Beaufsichtigung haben.

Es lässt sich festhalten, dass Banken und Versicherungen ein moderates - aber sehr heterogenes - Interesse an Regulierung haben und diese zur Schaffung eines »level playing field « nicht grundsätzlich ablehnen. Aufsicht hingegen stellt für alle Finanzinstitute einen zu minimierenden Kostenfaktor dar, sodass eine geringe Aufsichtsintensität und wohlwollende Aufsichtsbehörden bevorzugt werden. Dieses Wohlwollen lässt sich erreichen, indem Finanzunternehmen die Chance wahrnehmen, die BaFin als unabhängige Behörde zu vereinnahmen (agency capture). Ebenso ist vorstellbar, dass die Finanzbranche versucht, über politische Parteien und das BMF Einfluss auf die BaFin zu nehmen, wenn auf bilateraler Ebene keine Erfolge zu erwarten sind. Grundsätzlich sind Banken und Versicherungen jedoch an einem guten Kontakt zu den Aufsichtsbehörden BaFin und Bundesbank interessiert, da informale Abstimmungen die Kosten der aufsichtlichen Prüfung mindern können. Ebenso sind auch die Aufsichtsbehörden an der Kooperationsbereitschaft der überprüften Unternehmen interessiert, um personelle Ressourcen schonen und besser einsetzen zu können. So lässt sich resümieren, dass »die Effektivität der Aufsicht stärker von Konventionen und Normen abhängt als von formalen gesetzlichen Bestimmungen und dass die Möglichkeiten zur Einflussnahme durchaus subtil

107 Vgl. Kessler 2008, S. 52.

108 Freriks, Widmaier 2000, S. 109.

109 Vgl. Bergset et al. 2009; Osetrova 2007.

110 Vgl. Freriks, Widmaier 2000, S. 113. 
sind «. ${ }^{111}$ Das Interesse an einem kooperativen Umgang und die aufsichtsbezogenen Ziele aller übrigen Politikfeldakteure finden sich noch einmal zusammengefasst in der folgenden Übersicht.

Tabelle 1: Akteursziele im Politikfeld Finanzen

\begin{tabular}{|c|c|c|}
\hline $\begin{array}{l}\text { Zieldimension } \\
\text { Akteur }\end{array}$ & Steuerungsinteressen & $\begin{array}{l}\text { Ziel für institutionelle Finanz- } \\
\text { aufsicht }\end{array}$ \\
\hline $\mathrm{BMF}$ & $\begin{array}{l}\text { Umfassende Steuerung der Fi- } \\
\text { nanzaufsichtsbehörde; tatsächli- } \\
\text { che Eingriffe im Rahmen der } \\
\text { Fachaufsicht aber nur in Ausnah- } \\
\text { mefällen; besser: »vorauseilender } \\
\text { Gehorsam « des nachgeordneten } \\
\text { Bereichs }\end{array}$ & $\begin{array}{l}\text { Reibungslose Aufgabenerfül- } \\
\text { lung, d. h. konkretes Arrange- } \\
\text { ment der Institutionen ist zweit- } \\
\text { rangig }\end{array}$ \\
\hline BaFin & $\begin{array}{l}\text { Minimierung der Steuerungsein- } \\
\text { flüsse durch BMF; Versuch der } \\
\text { Emanzipation von der Fachauf- } \\
\text { sicht }\end{array}$ & $\begin{array}{l}\text { Weitgehende formale Unabhän- } \\
\text { gigkeit; Maximierung von perso- } \\
\text { nellen und finanziellen Ressour- } \\
\text { cen }\end{array}$ \\
\hline Bundesbank & $\begin{array}{l}\text { Vollständige Unabhängigkeit von } \\
\text { politischen Einflüssen }\end{array}$ & $\begin{array}{l}\text { Weitgehende formale Unabhän- } \\
\text { gigkeit; Maximierung von perso- } \\
\text { nellen und finanziellen Ressour- } \\
\text { cen }\end{array}$ \\
\hline $\begin{array}{l}\text { grabbing hand- } \\
\text { Parteien }\end{array}$ & $\begin{array}{l}\text { Maximale Zugriffsmöglichkeit } \\
\text { auf alle Behörden }\end{array}$ & $\begin{array}{l}\text { Fragmentierung; Vermeidung } \\
\text { von Machtkonzentrationen; Be- } \\
\text { friedigung von Klientelinteressen }\end{array}$ \\
\hline $\begin{array}{l}\text { helping hand- } \\
\text { Parteien }\end{array}$ & $\begin{array}{l}\text { Akzeptanz der Unabhängigkeit } \\
\text { und damit der teilweisen Unsteu- } \\
\text { erbarkeit von Behörden }\end{array}$ & $\begin{array}{l}\text { Einheitliche Struktur mit einer } \\
\text { starken Behörde; Befriedigung } \\
\text { von Gemeinwohlinteressen }\end{array}$ \\
\hline Finanzbranche & $\begin{array}{l}\text { »Capture «-Möglichkeit gegen- } \\
\text { über der Aufsichtsbehörde; Lob- } \\
\text { byismus gegenüber BMF und Par- } \\
\text { teien als indirekter Weg zur Ein- } \\
\text { flussnahme auf die Finanzaufsicht }\end{array}$ & $\begin{array}{l}\text { Fragmentierung der Aufsicht; ge- } \\
\text { ringe Eingriffsbefugnisse; wenig } \\
\text { fachlich herausragendes Perso- } \\
\text { nal; Aufsichtspersonal mit } \\
\text { Wechselinteresse in Richtung In- } \\
\text { dustrie }\end{array}$ \\
\hline
\end{tabular}

\section{Veränderungsmöglichkeiten in der Finanzmarktaufsicht}

Die Auseinandersetzung um die Reform der institutionellen Aufsicht folgt der Rationalität der Akteure im Politikfeld. Sowohl BaFin als auch Bundesbank streben den Fortbestand bzw. Ausbau ihrer jeweiligen Behörde an und bemühen sich um eine argumentative Untermauerung ihrer Position. Das CDU-geführte BMF und die Unionsparteien machen zum einen Gebrauch von ihrer Organisationskompetenz und bringen Reformvorschläge auf die politische Agenda, die Steuerungswillen be-

111 Ward 2002, S. 13.

Leviathan, 40. Jg., 1/2012 
weisen sollen. Zum anderen weist das Holding-Modell der Bundesbank den Weg zur grabbing hand-Politik, die nicht nur eine sich zu weit emanzipierende BaFin für ihre Entfernung von der politischen Steuerung » bestraft «, sondern auch eine Kompetenzbündelung der Aufsicht bei der Bundesbank vorsieht, die ein erstes Einfallstor für die ministerielle Fachaufsicht in diese Behörde öffnet. Diese Fachaufsicht würde nach dem Modell in allen Aufsichtsbereichen bestehen, weil die Schließung von Instituten als Maßnahme des Gewerbepolizeirechts interpretiert wird, die einer staatlichen Instanz bedarf (Art. 20 GG). Die Ablehnung des Integrationsmodells durch die CDU/CSU erklärt sich dagegen aus der Befürchtung eines vollständigen Steuerungsverlustes gegenüber der Aufsicht innerhalb einer unabhängigen Bundesbank. Die Eingliederung der BaFin in die Bundesbank würde eine starke und weisungsfreie Behörde entstehen lassen, die dem Ziel einer grabbing hand-Politik grundlegend widerspricht.

Die Interessen der Finanzbranche sind, wie gesagt, uneinheitlich, da Banken durchaus Vorteile darin sehen, dass sie nur noch von einer Behörde beaufsichtigt werden, während Versicherungen von der Bundesbank eine unsachgemäße und fachlich nicht ausreichend kompetente Aufsicht befürchten, die ihre Belange nur noch aus Sicht des Bankensektors berücksichtigen würde. ${ }^{112}$ Auch der Aspekt der informellen Zusammenarbeit zwischen Finanzunternehmen und Aufsicht spielt eine Rolle, da in der Vergangenheit seitens der Branche kaum Kritik an der BaFin laut wurde. Dies spricht für ein kooperatives Verhältnis, an das sich die Unternehmensseite gewöhnt hat und in dem sie einer Behörde gegenübersteht, die ihnen nur wenige Probleme bereitet. Die Veränderung dieses Zustands ist daher mit Unsicherheiten für die Zukunft verbunden und könnte möglicherweise zu höheren Kosten für die Branche führen. Damit lässt sich erklären, weshalb die Finanzbranche nicht offensiv auf den Umbau der institutionellen Aufsicht drängte.

Eine Intensivierung der Aufsichtstätigkeit und der Ausbau der BaFin oder einer anderen Aufsichtsbehörde würde nur dann umgesetzt werden, wenn sich die BaFin mit ihrem behördlichen Eigeninteresse im politischen Aushandlungsprozess durchsetzen könnte. Die Regierung steht einer Ausweitung der Aufsicht positiv oder zumindest neutral gegenüber, da einerseits »der Zielerreichungsgrad [der BaFin] - im negativen Sinne - daran gemessen wird, wie viele Bankenzusammenbrüche sie zulässt «, 113 und andererseits die Kosten für eine personelle Erweiterung durch die Umlagefinanzierung der Behörde nicht zulasten des Bundeshaushalts gehen. Banken und Versicherungen haben jedoch ein unverrückbares Interesse an schwacher Beaufsichtigung und werden sich mit hoher Wahrscheinlichkeit durchsetzen können, da vor allem die Bankenbranche eine große ökonomische Bedeutung hat. In der Vergangenheit zeigte sich daher, dass ein Zusammenhang zwischen der Bedeutung des Bankensektors und der Intensität der Aufsicht besteht, das heißt »je stärker der Bankensektor ist, desto nachsichtiger verhält sich die Aufsicht «. ${ }^{114}$ Die Berücksich-

112 Vgl. Fromme et al. 2010.

113 Kaserer 2006, S. 77.

114 Heinemann, Schüler 2004, S. 116. 
tigung von Brancheninteressen wird zudem durch die Strukturmerkmale der Aufsichtsbehörden selbst befördert. Wie kaum ein anderer Regulierungsbereich ist die Finanzmarktaufsicht mit einem besonderen Problem der Personalrekrutierung konfrontiert, das daraus entsteht, dass Finanzinstitute und Aufsichtsbehörden um Mitarbeiter mit derselben Qualifikation konkurrieren. Die BaFin bemüht sich dabei mit deutlich geringeren Ressourcen um Spitzenkräfte und ist häufig nur für Personen attraktiv, die stark intrinsisch motiviert sind oder aber extrinsisch motiviert und mittelmäßig befähigt. ${ }^{115}$ Letztere haben in der Regel weitergehende Karriereziele und streben einen Wechsel in die besser bezahlende Finanzbranche an, sobald sich dafür eine Gelegenheit bietet. ${ }^{116}$ Vor dem Hintergrund einer solchen Karrierestrategie entsteht für manche Mitarbeiter der Anreiz für ein funktional ungünstiges Wohlverhalten gegenüber den regulierten Unternehmen, das negative Effekte auf die Wirksamkeit der Aufsicht haben kann. Zu verhindern ist dies im bestehenden System allerdings nicht, sodass der Faktor Personal Gegenstand einer grundsätzlichen Reform sein sollte.

Es lässt sich festhalten, dass die Finanzmarktkrise zwar eine historische Chance für einige Akteure war, den Versuch einer Aufsichtsreform zu unternehmen. Allerdings konnte diese Chance nicht genutzt werden, da Gegner und Befürworter mit unvereinbaren Argumenten auf die Bundesregierung einwirkten. Nicht zu vergessen ist in diesem Zusammenhang auch, dass der Bund und die Länder selbst Eigentümer oder zumindest Anteilseigner großer Institute wie den Landesbanken oder der Hypo Real Estate sind, wodurch sich bei Akteuren dieser Körperschaften das Interesse an verschärfenden Veränderungen der Finanzaufsicht mit hoher Wahrscheinlichkeit abschwächt.

\section{Schlussbetrachtung}

Institutionelle Reformbestrebungen der Finanzmarktaufsicht wurden in diesem Beitrag als Funktion rationalen Handelns verschiedener Politikfeldakteure interpretiert. Selbst die Finanzkrise hat jedoch bislang nicht dazu geführt, dass Aufsicht oder Regulierung grundlegend neu konstruiert worden sind. Vielmehr stehen sich Akteure mit unveränderten Interessen gegenüber und versuchen sich in einem Aushandlungsprozess durchzusetzen. Die Finanzmarktkrise wurde zunächst als potentieller Auslöser für Veränderungen der Finanzmarktaufsicht und -regulierung begriffen, da sie auf den ersten Blick ein neues Problembewusstsein im Politikfeld geschaffen und den Boden für institutionelle Reformen bereitet hat. Eine genauere Betrachtung zeigt jedoch die strategischen und instrumentellen Beweggründe, die zu Reformvorschlägen und in dieser Konstellation auch zu ihrem Scheitern führten. Obwohl auf dem Höhepunkt der Krise beinahe alle Akteure entsprechende Veränderungen für sinnvoll hielten, hat es dennoch keine politische Fortentwicklung gegeben. Aufsicht und Regulierung sind derzeit auf einem ähnlichen Niveau wie 2008

115 Interviewnachweis 13.

116 Vgl. Ward 2002, S. 35.

Leviathan, 40. Jg., 1/2012 
und wurden nach den Erfahrungen mit der Finanzkrise nicht signifikant angepasst. Während sich die internationalen Regulierungsregime aufgrund der Uneinigkeit unter den Staaten kaum verschärften, blieben auch nationale Aufsichtsveränderungen aufgrund strategischer Akteursinteressen, die sich vor allem auf den Aspekt der ministeriellen Fachaufsicht über BaFin und Bundesbank kaprizierten, aus. Rationale, eigennutzorientierte Strategien von politischen Parteien, dem Finanzministerium, Marktteilnehmern und Aufsichtsbehörden führten zu einer Situation, in der sich die Positionen durchsetzten oder als gleich stark zeigten, die gegen eine intensivere Finanzaufsicht und eine starke Aufsichtsbehörde waren. Die Parteien CDU, CSU und FDP waren bemüht, Institutionen so umzugestalten, dass Machtkonzentrationen vermieden und Steuerungsmöglichkeiten erhöht würden. Die Oppositionsparteien schlossen sich dieser Strategie nicht an, sondern versuchten dagegen am Status quo festzuhalten. Beide Lager wurden dabei durch Positionen der Banken- bzw. Versicherungsbranche gestützt. Darüber hinaus konnten auch die Interessen von BaFin und Bundesbank nicht ohne weiteres übergangen werden, da beide Behörden durch ihr Renommee wichtige Positionen auf nationaler und internationaler Ebene haben und nur mit guten Begründungen zugunsten klientelistischer Überlegungen in ihren Kompetenzen beschnitten werden können. Da die Bundesbank einen nahezu unantastbaren Schutz ihrer Unabhängigkeit genießt, hätte nur die BaFin als politisches Opfer dienen und die Sündenbockfunktion für alles Versagen in der Finanzmarktkrise übernehmen können. Allerdings war selbst das BMF eher für eine Beibehaltung der bisherigen Aufsichtsstruktur, in der sich bereits stabile und funktionierende Interaktionsbeziehungen etabliert hatten. Die Interessen der einzelnen Akteure und Akteursgruppen waren hinsichtlich der Steuerung der behördlichen Aufsicht einerseits zwischen Gegnern und Befürwortern einer Reform und andererseits zwischen Strömungen innerhalb des Lagers der Befürworter gespalten. Dies führte zunächst zu einer Verschleppung der Aufsichtsreform und schließlich zum Scheitern eines institutionellen Politikwandels nach der Finanzkrise. Diese Rube nach dem Sturm wird auch in absehbarer Zukunft fortbestehen, da sich zum einen der akute Handlungsdruck reduziert hat und zum anderen die Akteursinteressen und -strategien durch die Krise keine Veränderung erfahren haben.

\section{Literatur}

Altvater, Elmar et al. 2010. Die Rückkehr des Staates? Nach der Finanzkrise. Hamburg: VSA Verlag.

Andolfatto, David 2004. Notes on The Grabbing Hand by Shleifer and Vishny. Working Paper, www.sfu.ca/ dandolfa/sv.pdf (Zugriff vom 28.12.2011).

Aulich, Chris; Batainah, Heba; Wettenhall, Roger 2010. "Autonomy and control in Australian agencies: data and preliminary findings from a cross-national empirical study «, in Australian Journal of Public Administration 69, 2, S. 214-228.

Arrow, Kenneth 1963. Social choice and individual values. New York: Wiley.

BaFin 2010 a. Mitarbeiter. http://www.bafin.de/cln_117/nn_722570/DE/BaFin/Organisation/M itarbeiter/mitarbeiter_node.html?_nnn=true (Zugriff vom 10.01.2012).

BaFin 2010 b. BaFin: Verwaltungsrat verabschiedet Haushaltsplan 2011.http://www.bafin.de/n n_2077192/SharedDocs/Artikel/DE/Service/Meldungen/2010/meldung_101125_verwrat __stellen.html (Zugriff vom 28.12.2011). 
Barth, James; Caprio, Gerard; Levine, Ross 2004. »Bank regulation and supervision: what works best? ", in Journal of Financial Intermediation 13, S. 205-248.

Bergset, Linda; Gebauer, Jana; Timme, Stephan 2009. "Branchenkriterien Banken ", in Anforderungen an die Nachbaltigkeitsberichterstattung: Kriterien und Bewertungsmethode im IÖW/future-Ranking, hrsg v. IÖW/future, S. 53-65. Berlin, Münster: IÖW/future e.V.

Berschens, Ruth 2009 .»EU-Finanzminister schwächen eigene Behörde «, in Wirtschaftswoche am 03.12.2009.

Beyers, Jan 2009. Multilevel venue-shopping in Europe. A comparative analysis of interest organizations in four EU member-states. EUSA Papers 2009. www.unc.edu/euce/eusa2009/ papers/beyers_05F.pdf (Zugriff vom 28.12.2011).

BMF 2011. Der Geschäftsbereich des BMF, Stand Dezember 2011. http://www.bundesfinanzm inisterium.de/nn_2998/DE/BMF__Startseite/Service/Downloads/Abt_Z/Uebersicht_Gesc haeftsbereich_BMF,templateId=raw, property=publicationFile.pdf (Zugriff vom 10.01.2012).

BMF 2010. Finanzmarktregulierung: Wie geht's voran? http://www.bundesfinanzministerium.d e/DE/Buergerinnen_und__Buerger/Gesellschaft_und_Zukunft/finanzkrise/20100730-Fi nanzmarktregulierung.html (Zugriff vom 28.12.2011).

BMI 2008. Grundsätze zur Ausübung der Fachaufsicht der Bundesministerien über den Geschäftsbereich. http://www.olev.de/f/DE-Bund_Fachaufsicht_grundsaetze_ausuebung_2008 -05-02.pdf (Zugriff vom 28.12.2011).

BMWi 2011. Jahreswirtschaftsbericht 2011: Deutschland im Aufschwung - den Wohlstand von morgen sichern. http://www.bmwi.de/BMWi/Redaktion/PDF/Publikationen/jahreswirtscha ftsbericht-2011, property=pdf,bereich=bmwi,sprache=de,rwb=true.pdf (Zugriff vom 29.01.2011).

Bundesrechnungshof 2005. Bericht an den Haushaltsausschuss des Deutschen Bundestages nach $\int 88$ Abs. 2 BHO zur Fachaufsicht der Bundesministerien über ihre nachgeordneten Geschäftsbereiche. Gz.: VII 4-2003-0396.

Clausen, Sven 2010. »Es lebe die Blutgrätsche «, in FTD vom 04.08.2010.

Cohen, Michael D.; March, James G.; Olsen, Johan P. 1972. »A garbage can model of organizational choice ", in Administrative Science Quarterly 17, 1, S. 1-25.

Commission of the European Communities 2009. Proposal for a regulation of the European Parliament and of the council establishing a European Insurance and Occupational Pensions Authority. COM(2009) 502 final. http://ec.europa.eu/internal_market/finances/docs/comm ittees/supervision/20090923/com2009_502_en.pdf (Zugriff vom 28.12.2011).

Czada, Roland 2003. "Grenzprobleme zwischen Politik und Markt", in Politik und Markt. Politische Vierteljahresschrift, Sonderheft 34, hrsg. v. Czada, Roland; Zintl, Reinhard, S. 9-26. Wiesbaden: VS Verlag für Sozialwissenschaften.

Dalla Pellegrina, Lucia; Masciandaro, Donato 2008. »Politicians, central banks, and the shape of financial supervision architectures ", in Journal of Financial Regulation and Compliance 16, 4, S. 290-317.

Dautzenberg, Leo; Kalb, Bartholomäus; Meister, Michael 2010. Koalition einig über Reform der nationalen Finanzaufsicht. Pressemitteilung der CDU/CSU, 16.12.2010. http://www.cducs u.de/Titel_pressemitteilung_koalition_einig_ueber_reform_der_nationalen_finanzaufsicht/ TabID_6/SubTabID_7/InhaltTypID_1/InhaltID_17465/Inhalte.aspx (Zugriff vom 28.12.2011).

Denters, Eric 2009. "Regulation and supervision of the global financial system ", in Amsterdam Law Forum 1, 3, S. 63-83.

Döhler, Marian 2002. "Institutional choice and bureaucratic autonomy in Germany ", in West European Politics 25, 1, S. 101-124.

Döhler, Marian 2006. "Regulative Politik und die Transformation der klassischen Verwaltung ", in Politik und Verwaltung. Auf dem Weg zu einer postmanagerialen Verwaltungsforschung. Politische Vierteljahresschrift, Sonderheft 37, hrsg. v. Bogumil, Jörg; Jann, Werner; Nullmeier, Frank, S. 208-227. Wiesbaden: VS Verlag für Sozialwissenschaften.

Döhler, Marian 2007. Die politische Steuerung der Verwaltung. Eine empirische Studie über politisch-administrative Interaktionen auf der Bundesebene. Staatslehre und politische Verwaltung, Bd. 11. Baden-Baden: Nomos. 
Döhler, Marian 2008. »Regieren mit Agenturen - Strategien und Interessen im exekutiven Binnenverhältnis ", in Regieren zu Beginn des 21. Jahrhunderts, hrsg. v. Jann, Werner; König, Klaus, S. 259-289. Tübingen: Mohr Siebeck.

Döhler, Marian 2011.»Wie Ministerien (versuchen,) Bundesbehörden (zu) steuern «, in Verwaltungsarchiv 102, S. 110-133.

Downs, Anthony 1967. Inside bureaucracy. Boston: Little, Brown and Company.

Dunleavy, Patrick 1991. Democracy, bureaucracy and public choice. Economic explanations in political science. London: Harvester.

Eberlein, Burkard 2000. »Regulierung und Konstitution von Märkten in Europa ", in Die politische Konstitution von Märkten, hrsg. v. Czada, Roland; Lütz, Susanne, S. 89-106. Wiesbaden: Westdeutscher Verlag.

Ebinger, Falk; Schmitt, Carina 2010. "Alles eine Frage des Managements? Wie Autonomierechte die Handlungsfreiheit des administrativen Führungspersonals beeinflussen ", in Politische Vierteljabresschrift 51, 1, S. 69-93.

Eichhorn, Peter et al. Hrsg. 1985. Verwaltungslexikon. Baden-Baden: Nomos.

Financial Stability Board 2009. Improving financial regulation-report by the FSB to G20 leaders. http://www.financialstabilityboard.org/publications/r_090925 b.pdf?frames=0 (Zugriff vom 10.01.2012).

Frach, Lotte. 2008. Finanzaufsicht in Deutschland und Großbritannien. Wiesbaden: VS Verlag für Sozialwissenschaften.

Freriks, Rainer; Widmaier, Ulrich 2000. »Die Veränderung der Strategie ökonomischer Akteure im Prozess der Entstehung eines europäischen Binnenmarktes ", in Die politische Konstitution von Märkten, hrsg. v. Czada, Roland; Lütz, Susanne, S. 107-123. Wiesbaden: Westdeutscher Verlag.

Fromme, Herbert; Tartler, Jens; Schrörs, Mark 2010. »Regierung vor Einigung zur Finanzaufsicht «, in FTD vom 18.03.2010.

Greive, Martin; Jost, Sebastian 2009. "Finanzaufsicht wird in Frankfurt konzentriert ", in Welt Online vom 09.10.2009. http://www.welt.de/die-welt/wirtschaft/article4783326/Finanzauf sicht-wird-in-Frankfurt-konzentriert.html (Zugriff vom 28.12.2011).

Hall, Peter; Taylor, Rosemary 1996. »Political science and the three new institutionalisms «, in Political Studies 44, S. 936-957.

Handke, Stefan 2010 a. »Ein konturloser Begriff mit Konjunktur: Das deutsche Verständnis von Finanzmarktregulierung ", in der moderne staat 3, 1, S. 53-69.

Handke, Stefan 2010 b. »Yes, we can (control them)! - Regulatory agencies: trustees or agents? «, in Goettingen Journal of International Law 2, 1, S. 111-134.

Hanke, Thomas 2009. "Der ausgelagerte Staat «, in Handelsblatt vom 28.05.2009.

Hartmann-Wendels, Thomas 2011.»Reform der Bankenaufsicht: Vertane Chance«, in Wirtschaftsdienst 91, 1, S. 4.

Heinemann, Friedrich; Schüler, Martin 2004. "A Stiglerian view on banking supervision ", in Public Choice 121, 1/2, S. 99-130.

Hood, Christopher; Rothenstein, Henry; Baldwin, Robert 2003. The government of risk. Oxford: Oxford University Press.

Hüther, Michael et al. 2009. Arbeitsweise der Bankenaufsicht vor dem Hintergrund der Finanzmarktkrise. http://www.iwkoeln.de/Portals/0/pdf/dokumente_andere/2009/Gutachten \%20 Bankenaufsicht.pdf (Zugriff vom 28.12.2011).

Kaiser, Stefan 2010. "Wie die Politik den Banken das Paradies erschafft", in FTD vom 18.05.2010.

Kaserer, Christoph 2006 .»Trends in der Bankenaufsicht als Motor der Überregulierung des Bankensektors - Anmerkungen aus einer politikökonomischen Perspektive ", in Perspektiven der Wirtschaftspolitik 7, 1, S. 67-87.

Kessler, Oliver 2008. Die internationale Politische Ökonomie des Risikos: Eine Analyse am Beispiel der Diskussion um die Reformierung der Finanzmärkte. Wiesbaden: VS Verlag für Sozialwissenschaften.

Koalitionsvertrag 2009. Wachstum, Bildung, Zusammenhalt. Koalitionsvertrag zwischen der CDU, CSU und FDP. www.cdu.de/doc/pdfc/091026-koalitionsvertrag-cducsu-fdp.pdf (Zugriff vom 28.12.2011).

Lane, Jan-Erik 1990. Institutional reform: a public policy perspective. Aldershot: Gower. 
Manager Magazin 2008. Regierung gibt Garantie für Spareinlagen. http://www.manager-maga zin.de/unternehmen/artikel/ 0,2828,582284,00.html (Zugriff vom 28.12.2011).

Mügge, Daniel 2010. "Conclusion: whither global financial governance after the crisis? «, in Global financial integration thirty years on, hrsg. v. Underhill, Geoffrey R. D. et al., S. 304-315. Cambridge: Cambridge University Press.

Niskanen, William 1979. "Ein ökonomisches Modell der Bürokratie«, in Ökonomische Theorie der Politik, hrsg. v. Pommerehne, Werner W.; Frey, Bruno S., S. 349-369. Berlin: Springer.

Osetrova, Anna 2007. Die Regulierung des Versicherungsmarkts unter besonderer Berücksichtigung der Solvabilitätsvorschriften. Karlsruhe: Verlag Versicherungswirtschaft.

Peters, Guy B. 2005. Institutional theory in political science. The new institutionalism. London: Continuum.

Posner, Elliot; Véron, Nicolas 2010. »The EU and financial regulation: power without purpose? «, in Journal of European Public Policy 17, 3, S. 400-415.

Quintyn, Marc; Taylor, Michael W. 2004. Should financial sector regulators be independent? Economic Issues 32. Washington D.C.: International Monetary Fund.

Rude, Christopher 2008. "The global financial crisis: what needs to be done? ", in FES Briefing Paper 12, November 2008.

Sanio, Jochen 2002. Die Versicherungsaufsicht nach Errichtung der BaFin. Vortrag von Jochen Sanio anlässlich der Mitgliederversammlung des Gesamtverbandes der Deutschen Versicherungswirtschaft e.V. (GDV) am 14. November 2002 in Berlin. http://www.bafin.de/nn_99 2414/SharedDocs/Reden/DE/2002/re_021114_sanio.html (Zugriff vom 28.12.2011).

Shleifer, Andrei; Vishny, Robert W. 1999. The grabbing hand. Government pathologies and their cures. Cambridge: Harvard University Press.

Stigler, George 1971. "The theory of economic regulation ", in Bell Journal of Economics and Management Science 2, S. 3-21.

Sturm, Roland et al. 2002. Der regulatorische Staat: Deutschland und Großbritannien im Vergleich. London: Anglo-German Foundation.

van der Heijden, Jeroen 2011. "Institutional layering: a review of the use of the concept ", in Politics 31, 1, S. 9-18.

Wallstreet online 2010. Finanzaufsicht - Holding-Modell: Regierung kurz vor Einigung, 19.03.2010. http://www.wallstreet-online.de/nachrichten/nachricht/2914030-finanzaufsich t-holding-modell-regierung-kurz-vor-einigung.html (Zugriff vom 28.12.2011).

Ward, Jonathan 2002. The supervisory approach: a critique. Working paper. Cambridge: Cambridge Endowment for Research in Finance.

Weiland, Severin; Goetz, John 2009. »Union rückt von Staatssekretär Asmussen ab «, in: Spiegel online, 25.06.2009. http://www.spiegel.de/politik/deutschland/0,1518,631710,00.html (Zugriff vom 19.10.2010).

Wissenschaftlicher Beirat beim BMWi 2010. Reform der Bankenaufsicht nach der Finanzkrise. Gutachten vom 16. April 2010.

Zahariadis, Nikolaos 2007. The multiple streams framework: structure, limitations, prospects, in Theories of the policy process, hrsg. v. Sabatier, Paul, S. 65-92. Boulder: Westview Press. 
Zitierte Interviews aus dem Jahr 2010

Anonym, ehemaliger Mitarbeiter im Leitungsbereich der BaFin, derzeitige Beschäftigung in leitender Funktion bei einem deutschen Versicherungsunternehmen, 13.07.2010.

Binding, Lothar, Mitglied des Deutschen Bundestags, SPD-Fraktion, Mitglied des Finanzausschusses, 01.07.2010.

Dautzenberg, Leo, Mitglied des Deutschen Bundestags, CDU/CSU-Fraktion, Mitglied des Finanzausschusses, 22.06.2010.

Quinten, Daniel, Deutsche Bundesbank, Büroleiter des Vizepräsidenten Franz-Christoph Zeitler, 11.10.2010.

Sell, Michael, Bundesanstalt für Finanzdienstleistungsaufsicht, Exekutivdirektor Querschnittsaufgaben/Innere Verwaltung, 30.08.2010.

Troost, Axel, Mitglied des Deutschen Bundestags, Fraktion DIE LINKE, Mitglied des Finanzausschusses, 06.07.2010.

Wehling, Axel, Gesamtverband der Deutschen Versicherungswirtschaft, Mitglied der Hauptgeschäftsführung, 08.10.2010.

Wissing, Volker, Mitglied des Deutschen Bundestags, FDP-Fraktion, Vorsitzender des Finanzausschusses, 15.07.2010. 
Zusammenfassung: Reformen der institutionellen Finanzmarktaufsicht waren nach der Finanzkrise 2008 durch politikfeldspezifische Akteurskonstellationen und Nutzenüberlegungen determiniert. Am Beispiel der deutschen Debatte lassen sich die Strategien von politischen Akteuren, Marktteilnehmern und Verwaltungsbehörden im Rahmen einer Public ChoiceModellierung darstellen, die verdeutlicht, welche Interessen hinsichtlich der Ausgestaltung verschiedener Aufsichtsmodelle bestehen. Im Mittelpunkt steht dabei einerseits das Bestreben von klientelistisch ausgerichteten Parteien und des Finanzministeriums, die Bundesanstalt für Finanzdienstleistungsaufsicht (BaFin) nach den eigenen Vorstellungen kontrollieren und steuern zu können. Andererseits stehen dem die gegenläufigen Bemühungen von gemeinwohlorientierten Parteien, der BaFin und der Deutschen Bundesbank gegenüber. Institutionelle Reformvorschläge, die kurzfristig durch die Finanzmarktkrise auf die politische Agenda gebracht worden sind, treffen damit auf langfristig stabile Akteurspräferenzen. Diese Konfliktsituation, in der nicht nur unterschiedliche Steuerungsinteressen, sondern auch Kostenüberlegungen für das Gut der Aufsicht eine Rolle spielen, verhinderte eine Veränderung des institutionellen Status quo. Die Chance zu einer Reform der deutschen Finanzmarktaufsicht, die sich durch die Krise eröffnet hatte, blieb daher ungenutzt.

Schlagwörter: Deutsche Finanzmarktaufsicht, BaFin, Finanzmarktkrise, Institutionenwandel

\section{Never waste a good crisis - political struggles and the lost opportunity for institutional reform in German financial market supervision}

Summary: The financial crisis of 2008 revealed weaknesses in the policymaking and supervisory capacities of the German Ministry of Finance (BMF) and the German Financial Market Supervisory Authority (BaFin). In the aftermath of the crisis institutional reforms have been discussed on the political level; however, the actors' strategies in German financial market politics have led to a kind of deadlock which has prevented policy change. Utility maximizing organizations, political parties, and business enterprises have all had different interests in terms of regulation, supervision, and the institutional design of supervising authorities. This is why, after the crisis, institutional change and improvements infinancial market supervision in Germany have yet to come to fruition.

Keywords: German financial supervision, BaFin, financial crisis, institutional change

Autor

Stefan Handke

Leibniz Universität Hannover

Institut für Politische Wissenschaft

Schneiderberg 50

30167 Hannover

st.handke@ipw.uni-hannover.de

Leviathan, 40. Jg., 1/2012 